\title{
GLOBAL ATTRACTIVITY IN AN RBC SURVIVAL MODEL OF WAZEWSKA AND LASOTA
}

\author{
BY \\ JING-WEN LI (Department of Mathematics, Shaoyang Normal College, Shaoyang, Hunan, \\ 422000, P. R. China) \\ AND \\ SUI SUN CHENG (Department of Mathematics, Tsing Hua University, Hsinchu, Taiwan, 30043, \\ R. O. C.)
}

\begin{abstract}
We obtain a condition for the positive equilibrium to be a global attractor of the survival model of red blood cells proposed by Wazewska and Lasota. Our technique is novel in the sense that a pair of nonlinear equations is utilized, and our result improves earlier results in [3] and [4].
\end{abstract}

1. Introduction. A well-known model for the survival of red blood cells was proposed by Wazewska-Czyzewska and Lasota in 1976 [1] and stated in terms of a nonlinear ordinary differential equations with a delayed parameter:

$$
N^{\prime}(t)=-\mu N(t)+p e^{-\gamma N(t-\tau)}, \quad t \geq 0,
$$

where $\mu \in(0,1)$ and $\gamma, p, \tau \in(0, \infty)$. Here $N(t)$ denotes the number of red blood cells at time $t, \mu$ is the probability of death of a red blood cell, $p$ and $\gamma$ are positive constants related to the production of red blood cells per unit time, and $\tau$ is the time required to produce a red blood cell.

The properties of this equation are of interest from both a mathematical and a biological point of view. In particular, one main concern is when the equilibrium will become a global attractor of solutions defined by "positive initial conditions". More precisely, let

$$
\Omega=\{\phi \in C([-\tau, 0],[0, \infty)) \mid \phi(0)>0\} .
$$

The solution $N=N(t)$ of (1) defined by the initial condition $N(t)=\phi(t)$ for $t \in[-\tau, 0]$ exists, is unique and is positive for $t \geq 0$ by means of the standard method of steps (see, e.g., [2, p. 89]). As an example, a constant solution $N(t)=N^{*}$ can be found and is given by the unique positive solution of the transcendental equation

$$
\mu N^{*}=p e^{-\gamma N^{*}} \text {. }
$$

Received July 5, 2000.

2000 Mathematics Subject Classification. Primary 92C30, 92D25.

Key words and phrases. Delay differential equation, Wazewska-Lasota model, red blood cell, global attractivity. 
This solution is called the equilibrium solution and the number $N^{*}$ is called the (positive) equilibrium point of (1). If $N^{*}$ has the property that for any solution $N(t)$ of (1) that satisfies $N(t)=\phi(t)$ for $t \in[-\tau, 0]$, we have $\lim _{t \rightarrow \infty} N(t)=N^{*}$, then $N^{*}$ is said to be a global attractor of $(1)$ for solutions originating from $\Omega$.

There are a number of results that tell us when $N^{*}$ is a global attractor. For example, in [3], it is shown that if

$$
\gamma N^{*}\left(1-e^{-\mu \tau}\right)<\ln 2
$$

then $N^{*}$ is a global attractor. As an improvement, it is shown in [4] that the same conclusion will hold even if the number $\ln 2$ in the above condition can be replaced by the larger number 1 . The question then arises as to whether sharper conditions can be found. In this paper, we will address this relatively difficult problem and show that the improved condition

$$
\gamma N^{*}\left(1-e^{-\mu \tau}\right) \leq 1+\frac{e^{-\mu \tau}}{\gamma N^{*}}
$$

is sufficient for $N^{*}$ to be a global attractor for solutions originating from $\Omega$.

2. Auxiliary inequalities. Our investigations lead to the following coupled pair of inequalities:

$$
\begin{aligned}
& y+e^{-\mu \tau} \ln \left(1+\frac{y}{\gamma N^{*}}\right) \leq M\left(e^{-x}-1\right), \\
& x+e^{-\mu \tau} \ln \left(1+\frac{x}{\gamma N^{*}}\right) \geq M\left(e^{-y}-1\right),
\end{aligned}
$$

where we have set

$$
M \equiv \gamma N^{*}\left(1-e^{-\mu \tau}\right)
$$

for the sake of convenience. Clearly, $(x, y)=(0,0)$ is a solution of $(3)-(4)$. We will need the fact that there are no nontrivial solutions of $(3)-(4)$ in the region

$$
\Phi=\left\{(x, y) \mid-\gamma N^{*}<x \leq 0 \leq y<\infty\right\} .
$$

Theorem 1. If $(2)$ holds, then $(0,0)$ is the only solution of $(3)-(4)$ in $\Phi$.

Proof. There are three cases to consider: $M \leq 1,1<M<1+e^{-\mu \tau} /\left(\gamma N^{*}\right)$, and $M=1+e^{-\mu \tau} /\left(\gamma N^{*}\right)$. Suppose first that $M \leq 1$. Let $(\alpha, \beta)$ be a solution of $(3)-(4)$ in $\Phi$. Then in view of $M \leq 1$,

$$
\begin{aligned}
& \beta \leq \beta+e^{-\mu \tau} \ln \left(1+\frac{\beta}{\gamma N^{*}}\right) \leq M\left(e^{-\alpha}-1\right) \leq e^{-\alpha}-1, \\
& \alpha \geq \alpha+e^{-\mu \tau} \ln \left(1+\frac{\alpha}{\gamma N^{*}}\right) \geq M\left(e^{-\beta}-1\right) \geq e^{-\beta}-1,
\end{aligned}
$$

so that

$$
\beta \leq \exp \left(1-e^{-\beta}\right)-1 .
$$

Note, however, that the function

$$
h(t)=\exp \left(1-e^{-t}\right)-1-t, \quad t \geq 0,
$$


satisfies $h(0)=0$,

$$
h^{\prime}(t)=e^{-t} \exp \left(1-e^{-t}\right)-1
$$

and

$$
h^{\prime \prime}(t)=e^{-t}\left(e^{-t}-1\right) \exp \left(1-e^{-t}\right)<0
$$

for $t>0$. Thus $h^{\prime}(t)<h^{\prime}(0)=0$ for $t>0$, which shows that $h(t)$ cannot have any root in $(0, \infty)$. As a consequence, (5) can only hold for $\beta=0$. Similarly, we may show that $\alpha=0$.

Next we consider the case where $1<M<1+e^{-\mu \tau} /\left(\gamma N^{*}\right)$. Let functions $f$ and $g$ be defined by

$$
f(t)=t+e^{-\mu \tau} \ln \left(1+\frac{t}{\gamma N^{*}}\right), \quad t>-\gamma N^{*}
$$

and

$$
g(t)=M\left(e^{-t}-1\right), \quad t \in R,
$$

respectively. Then (3) can be written as

$$
f(y) \leq g(x)
$$

while (4) can be written as

$$
f(x) \geq g(y)
$$

We now show that the implicit relation $f(y)=g(x)$ in $\Phi$ defines a function $y=C_{1}(x)$ for $-\gamma N^{*}<x \leq 0$, and the implicit relation $f(x)=g(y)$ in $\Phi$ defines a function $y=C_{2}(x)$ for $x$ in some interval of the form $\left(x^{*}, 0\right]$ where $-\gamma N^{*}<x^{*}<0$. To see this, note that $f(0)=0, \lim _{t \rightarrow-\gamma N^{*}} f(t)=-\infty, \lim _{t \rightarrow \infty} f(t)=+\infty$,

$$
f^{\prime}(t)=1+\frac{e^{-\mu \tau}}{\gamma N^{*}+t}>0, \quad t>-\gamma N^{*},
$$

and

$$
f^{\prime \prime}(t)=\frac{-e^{-\mu \tau}}{\left(\gamma N^{*}+t\right)^{2}}<0, \quad t>-\gamma N^{*} .
$$

Thus $f$ is a strictly increasing and strictly concave function defined on $\left(-\gamma N^{*}, \infty\right)$ and its range is $R$. In particular, the inverse $f^{-1}$ exists and is defined on $R$. This shows that $C_{1}(x)=f^{-1}(g(x))$ for $-\gamma N^{*}<x \leq 0$. Similarly, $g(0)=0, \lim _{t \rightarrow-\infty} g(t)=+\infty$, $\lim _{t \rightarrow \infty} g(t)=-M$,

$$
g^{\prime}(t)=-M e^{-t}<0, \quad t \in R
$$

and

$$
g^{\prime \prime}(t)=M e^{-t}>0, \quad t \in R
$$

This shows that the inverse $g^{-1}$ exists on $(-M,+\infty)$. Note that the properties of $f$ guarantee that there is some point $x^{*}$ in $\left(-\gamma N^{*}, 0\right)$ such that $f\left(x^{*}\right)=-M$ and $f(x)>$ $-M$ for $x>x^{*}$. Thus $C_{2}(x)=g^{-1}(f(x))$ for $x^{*}<x \leq 0$. Both graphs of the functions 
$C_{1}$ and $C_{2}$ pass through the point $(0,0)$ since $f(0)=g(0)=0$. They lie entirely in $\Phi$ since by means of implicit differentiation,

$$
C_{1}^{\prime}(x)=\frac{g^{\prime}(x)}{f^{\prime}(y)}<0, \quad(x, y) \in \Phi,
$$

and

$$
C_{2}^{\prime}(x)=\frac{f^{\prime}(x)}{g^{\prime}(y)}<0, \quad(x, y) \in \Phi .
$$

Furthermore, the graph of $C_{2}$ lies above the straight line defined by $y=-x$, since $C_{2}^{\prime}(0)<-1$ and

$$
C_{2}^{\prime \prime}(x)=\frac{1}{M e^{-y}} \frac{e^{-\mu \tau}}{\left(\gamma N^{*}+x\right)^{2}}+\left(C_{2}^{\prime}(x)\right)^{2}>0, \quad x^{*}<x<0 .
$$

Next, we assert that they do not intersect each other in $\Phi$ except at the point $(0,0)$. To see this, it suffices to show that $C_{2}(x)>C_{1}(x)$ for $x^{*}<x<0$. Indeed, note that $C_{1}(0)=C_{2}(0)=0$, and

$$
0>C_{1}^{\prime}(0)=-M \frac{1}{\left(1+e^{-\mu \tau} /\left(\gamma N^{*}\right)\right)}>-1>-\frac{1}{M}\left(1+e^{-\mu \tau} /\left(\gamma N^{*}\right)\right)=C_{2}^{\prime}(0)
$$

due to our assumption $M<1+e^{-\mu \tau} /\left(\gamma N^{*}\right)$; thus $C_{2}(x)>-x>C_{1}(x)$ for $x$ in a left neighborhood of 0 . Assume to the contrary that the graphs $C_{1}$ and $C_{2}$ intersect in $\Phi$. Then there must be some point $(\tilde{x}, \tilde{y})$ of intersection in $\Phi$ such that $\tilde{x} \in\left(x^{*}, 0\right), C_{2}(x)>$ $C_{1}(x)$ for $x \in(\tilde{x}, 0)$,

$$
C_{2}(\tilde{x})=C_{1}(\tilde{x})=\tilde{y}>-\tilde{x}>0,
$$

and

$$
\frac{C_{2}^{\prime}(\tilde{x})}{C_{1}^{\prime}(\tilde{x})}=\lim _{t \rightarrow 0^{+}} \frac{C_{2}(\tilde{x})-C_{2}(\tilde{x}+t)}{C_{1}(\tilde{x})-C_{1}(\tilde{x}+t)} \leq 1
$$

However, note that

$$
\begin{aligned}
\frac{C_{2}^{\prime}(\tilde{x})}{C_{1}^{\prime}(\tilde{x})} & =\frac{f^{\prime}(\tilde{x})}{g^{\prime}(\tilde{y})} \frac{f^{\prime}(\tilde{y})}{g^{\prime}(\tilde{x})} \\
& =\frac{1}{M^{2}}\left(1+\frac{e^{-\mu \tau}}{\gamma N^{*}+\tilde{x}}\right)\left(1+\frac{e^{-\mu \tau}}{\gamma N^{*}+\tilde{y}}\right) e^{\tilde{x}+\tilde{y}}
\end{aligned}
$$

Therefore, there will be a contradiction if we can show that the function

$$
\Psi(u)=\left(1+\frac{e^{-\mu \tau}}{\gamma N^{*}+\tilde{x}}\right)\left(1+\frac{e^{-\mu \tau}}{\gamma N^{*}+u}\right) e^{\tilde{x}+u}, \quad u \geq-\tilde{x},
$$

is increasing and $\Psi(-\tilde{x}) \geq M^{2}$. The required properties of $\Psi$ can easily be seen. Indeed,

$$
\begin{aligned}
\Psi(-\tilde{x}) & =\left(1+\frac{e^{-\mu \tau}}{\gamma N^{*}+\tilde{x}}\right)\left(1+\frac{e^{-\mu \tau}}{\gamma N^{*}-\tilde{x}}\right) \\
& \geq\left(1+\frac{e^{-\mu \tau}}{\gamma N^{*}}\right)^{2} \geq M^{2} .
\end{aligned}
$$


Furthermore,

$$
\Psi^{\prime}(u)=\left(1+\frac{e^{-\mu \tau}}{\gamma N^{*}+\tilde{x}}\right)\left(1+\frac{e^{-\mu \tau}}{\gamma N^{*}+u}-\frac{e^{-\mu \tau}}{\left(\gamma N^{*}+u\right)^{2}}\right) e^{\tilde{x}+u} .
$$

But since $M=\gamma N^{*}\left(1-e^{-\mu \tau}\right)>1$, we see that

$$
1<\frac{1}{\gamma N^{*}}<1-e^{-\mu \tau}
$$

which implies

$$
\frac{e^{-\mu \tau}}{\left(\gamma N^{*}+u\right)^{2}} \leq \frac{e^{-\mu \tau}}{\left(\gamma N^{*}\right)^{2}}<\frac{e^{-\mu \tau}}{\gamma N^{*}}<e^{-\mu \tau}\left(1-e^{-\mu \tau}\right) \leq \frac{1}{4}
$$

and

$$
\Psi^{\prime}(u) \geq\left(1+\frac{e^{-\mu \tau}}{\gamma N^{*}+\tilde{x}}\right)\left(\frac{e^{-\mu \tau}}{\gamma N^{*}+u}+\frac{3}{4}\right) e^{\tilde{x}+u}>0, \quad u \geq-\tilde{x},
$$

as required. Finally, note that a point $(x, y) \in \Phi$ satisfies $f(x) \geq g(y)$ if, and only if, it lies above or on the graph $C_{2}$, and it satisfies $f(y) \leq g(x)$ if, and only if, it lies below or on the graph $C_{1}$. Since we have just shown that $C_{1}$ and $C_{2}$ intersect each other only at $(0,0)$, thus the only $(x, y) \in \Phi$ that satisfies $(3)-(4)$ is $(0,0)$. The proof of the second case is complete.

As for the last case where $M=1+e^{-\mu \tau} /\left(\gamma N^{*}\right)$, we define $f, g, C_{1}$ and $C_{2}$ as in the previous case. Here, however, we may check that $C_{1}(0)=C_{2}(0)=0, C_{1}^{\prime}(0)=C_{2}^{\prime}(0)=$ $-1$

$$
C_{1}^{\prime \prime}(0)=C_{2}^{\prime \prime}(0)=1+\frac{1}{M} \frac{e^{-\mu \tau}}{\left(\gamma N^{*}\right)^{2}}
$$

and

$$
C_{2}^{\prime \prime \prime}(0)-C_{1}^{\prime \prime \prime}(0)=\frac{3}{M^{2}}\left(\frac{e^{-\mu \tau}}{\left(\gamma N^{*}\right)^{2}}\right)^{2}-1 .
$$

Since $M>1$, the inequalities in (6) hold, so that

$$
C_{2}^{\prime \prime \prime}(0)-C_{1}^{\prime \prime \prime}(0)<3 \frac{1}{4^{2}}-1<0 .
$$

Accordingly, by expressing $C_{2}(x)-C_{1}(x)$ in the form of a third-order polynomial about 0 plus a remainder term of the form $o\left(x^{3}\right)$, we see that $C_{2}(x)>C_{1}(x)$ for $x$ in a left neighborhood of 0 . By the same arguments as shown above, we then see that $C_{2}(x)>$ $C_{1}(x)$ for $x^{*}<x<0$. This implies that $(0,0)$ is the only solution of $(3)-(4)$ in $\Phi$. The proof is complete.

3. Proof of main result. The change of variables

$$
N(t)=N^{*}+\frac{1}{\gamma} x(t)
$$

reduces Eq. (1) to the delay differential equation

$$
x^{\prime}(t)+\mu x(t)+\gamma \mu N^{*}\left(1-e^{-x(t-\tau)}\right)=0, \quad t \geq 0 .
$$


Thus, in order to show that every solution of (1) that is positive for $t \geq 0$ will converge to $N^{*}$, it suffices to show that every solution $x(t)$ of $(7)$ that satisfies $x(t) \geq-\gamma N^{*}$ for $t \geq 0$ will converge to zero.

In order to see this, we need to consider three kinds of solutions. The first kind consists of positive solutions, the second negative solutions, and the third oscillatory solutions. It has already been shown (see, e.g., $[2,4]$ ) that if $x=x(t)$ is a solution of (7) that is eventually positive or eventually negative, then $x(t) \rightarrow 0$ as $t$ tends to $\infty$. It has also been shown [3] that if $x=x(t)$ is an oscillatory solution of $(7)$, then $|x(t)| \leq M$ for all large $t$. Thus, if $x(t)$ is an oscillatory solution of (7) such that $x(t) \geq-\gamma N^{*}$ for $t \geq 0$, then letting

$$
x_{-}=\liminf _{t \rightarrow \infty} x(t)
$$

and

$$
x_{+}=\limsup _{t \rightarrow \infty} x(t)
$$

we see that

$$
-\gamma N^{*}<-M \leq x_{-} \leq 0 \leq x_{+}<\infty
$$

We assert further that

$$
x_{+}+e^{-\mu \tau} \ln \left(1+\frac{x_{+}}{\gamma N^{*}}\right) \leq M\left(e^{-x_{-}}-1\right)
$$

and

$$
x_{-}+e^{-\mu \tau} \ln \left(1+\frac{x_{-}}{\gamma N^{*}}\right) \geq M\left(e^{-x_{+}}-1\right) .
$$

To see this, let $\varepsilon>0$ and $T>0$ be such that

$$
x_{+}-\varepsilon<x(t)<x_{+}+\varepsilon, \quad t \geq T+\tau .
$$

Further, let $\left\{t_{n}\right\}_{n=1}^{\infty}$ be a positive, increasing, and divergent sequence of real numbers such that $x^{\prime}\left(t_{n}\right)=0$ for $n \geq 1$, and $\lim _{n \rightarrow \infty} x\left(t_{n}\right)=x_{+}$. Then in view of $(7)$,

$$
x\left(t_{n}\right)+\gamma N^{*}\left(1-e^{x\left(t_{n}-\tau\right)}\right)=0,
$$

so that

$$
x\left(t_{n}-\tau\right)=-\ln \left(1+\frac{x\left(t_{n}\right)}{\gamma N^{*}}\right) .
$$

On the other hand, in view of (7),

$$
\left(x(t) e^{\mu t}\right)^{\prime}=\gamma \mu N^{*}\left(e^{-x(t-\tau)}-1\right) e^{\mu t}<\gamma \mu N^{*}\left(e^{-x_{-}+\varepsilon}-1\right) e^{\mu t},
$$

which yields, after integrating from $t_{n}-\tau$ to $t_{n}$,

$$
x\left(t_{n}\right)-x\left(t_{n}-\tau\right) e^{-\mu \tau} \leq \gamma N^{*}\left(e^{-x_{-}+\varepsilon}-1\right)\left(1-e^{-\mu \tau}\right) .
$$

Substituting (10) into the above expression, we see that

$$
x\left(t_{n}\right)+\ln \left(1+\frac{x\left(t_{n}\right)}{\gamma N^{*}}\right) e^{-\mu \tau} \leq M\left(e^{-x_{-}+\varepsilon}-1\right) .
$$


By taking limits on both sides as $n \rightarrow \infty$ and then as $\varepsilon \rightarrow 0$, we see that (8) will follow as required. The inequality (9) is similarly proved.

We now invoke Theorem 1 and conclude that $x_{-}=x^{+}=0$. The formal result now follows.

ThEOREM 2. Let $N^{*}$ be the unique positive solution of the equation $\mu N^{*}=p e^{-\gamma N^{*}}$. Suppose

$$
\gamma N^{*}\left(1-e^{-\mu \tau}\right) \leq 1+\frac{e^{-\mu \tau}}{\gamma N^{*}}
$$

Then for every solution $N=N(t)$ of (1) that is positive on $[0, \infty), \lim _{t \rightarrow \infty} N(t)=N^{*}$.

We remark that the condition (11) is nonvacuous in the sense that there are $\mu \in(0,1)$, $\gamma, p, \tau \in(0, \infty)$ such that the equality sign in (11) is satisfied. Indeed, let $\mu, \gamma$ and $p$ satisfy

$$
(\sqrt{2}+1) e^{\sqrt{2}+1}=\frac{\gamma p}{\mu}
$$

and let $\tau$ satisfy $e^{-\mu \tau}=1 / 2$. Then $\gamma N^{*}=\sqrt{2}+1$, so that

$$
\gamma N^{*}\left(1-e^{-\mu \tau}\right)=\frac{\sqrt{2}+1}{2}=1+\frac{e^{-\mu \tau}}{\gamma N^{*}},
$$

as required.

\section{REFERENCES}

[1] M. Wazewska-Czyzewska and A. Lasota, Mathematical problems of the dynamics of a system of red blood cells, Mat. Stos. (3) 6, 23-40 (1976) (Polish)

[2] I. Györi and G. Ladas, Oscillation Theory of Delay Differential Equations with Applications, Clarendon Press, Oxford, 1991

[3] M. R. Kulenovic, G. Ladas, and Y. G. Sficas, Global attractivity in population dynamics, Computers and Math. Applic. 18, 925-928 (1989)

[4] J. W. Li, Asymptotic behavior of delay differential model, J. Biomath. 9, 91-95 (1994) 